\title{
Hyponatremia and poor cognitive outcome following pediatric brain tumor surgery
}

\author{
Cydni N. Williams, MD, ${ }^{1}$ Jay Riva-Cambrin, MD, MSc, ${ }^{2}$ Angela P. Presson, $\mathrm{PhD},{ }^{3}$ and \\ Susan L. Bratton, MD, MPH ${ }^{4}$
}

1Department of Pediatrics, Oregon Health \& Science University, Portland, Oregon; ${ }^{2}$ Department of Neurosurgery, ${ }^{3}$ Division of Epidemiology, Department of Internal Medicine, and ${ }^{4}$ Department of Pediatrics, University of Utah, Salt Lake City, Utah

\begin{abstract}
OBJECT Pediatric intracranial neoplasms are common and cause substantial neurological morbidity. Postoperative hyponatremia is also common and may exacerbate neurological injury. The authors performed an exploratory analysis to evaluate an exposure-response relationship between hyponatremia severity and cognitive function at discharge.
\end{abstract}

METHODS A retrospective cohort of patients 0-19 years old who underwent a first intracranial neoplasm surgery at a pediatric tertiary care hospital was reviewed. Outcome was assessed by Pediatric Cerebral Performance Category (PCPC) score of 1-6 at hospital discharge. Poor outcome was defined as PCPC score 3-6, corresponding to moderate or worse disability.

RESULTS Of 319 total children, $80(25 \%)$ had poor outcomes. One hundred thirty-seven children (43\%) had serum sodium concentrations $\leq 131-135 \mathrm{mEq} / \mathrm{L}$ and $39(12 \%)$ had serum sodium concentrations $\leq 130 \mathrm{mEq} / \mathrm{L}$. Lower nadir sodium concentration and longer duration of hyponatremia were significantly associated with worsening PCPC score ( $p$ $<0.001)$. Rapid sodium decreases and more hyponatremic episodes were also significantly associated with worsening PCPC score ( $p$ < 0.001). After adjustment for patient factors, tumor characteristics, and measures of sodium disruption, multivariable analysis revealed noncortical tumor locations and lower nadir sodium concentration (adjusted odds ratio 0.86, 95\% confidence interval 0.78-0.95) were important independent risk factors for poor cognitive outcome.

CONCLUSIONS Neurocognitive disability and hyponatremia are common in children undergoing surgery for intracranial neoplasms. This study found a significant association between severity of hyponatremia and worsened cognitive outcome, with an apparent exposure-response relationship. These data support the need for careful postoperative monitoring of serum sodium. Further research is needed to determine if prevention and treatment of hyponatremia can improve outcomes in these children.

http://thejns.org/doi/abs/10.3171/2014.10.PEDS14368

KEY WORDS hyponatremia; oncology; serum sodium; intracranial neoplasm; cognitive function

I NTRACRANIAL neoplasms account for nearly $25 \%$ of pediatric cancers ${ }^{32}$ and $60 \%-70 \%$ of children who survive are left with disabilities contributing to long-term morbidity. 1,7,17,21,22 Most children diagnosed with intracranial neoplasms undergo at least 1 neurosurgical procedure for diagnosis and treatment. Hyponatremia following neurosurgical procedures in children with brain tumors is common, with mild sodium derangement occurring in half of the children ${ }^{4}$ and severe sodium derangement occurring in approximately $12 \% .4,30,31$ In some clinical settings, hy- ponatremia is known to exacerbate cerebral edema and cause seizures and encephalopathy, which can contribute to secondary brain injury following neurosurgery. ${ }^{19,26,28}$

Young age, hydrocephalus, malignant histology, and noncortical tumor location are important clinical risk factors for hyponatremia in the postoperative period among children with intracranial neoplasms..$^{30,31}$ These features overlap with some of the patient factors, tumor-related characteristics, and treatments (such as age, histology, hydrocephalus, tumor location, extent of resection, reop-

ABBREVIATIONS $\mathrm{Cl}=$ confidence interval; ICD-9-CM = International Classification of Diseases, Ninth Revision, Clinical Modification; IQR = interquartile range; OR = odds ratio; $\mathrm{PCPC}=$ Pediatric Cerebral Performance Category; VIF = variance inflation factor.

SUBMITTED July 15, 2014. ACCEPTED October 31, 2014.

INCLUDE WHEN CITING Published online February 27, 2015; DOI: 10.3171/2014.10.PEDS14368.

DISCLOSURE The authors report no conflict of interest concerning the materials or methods used in this study or the findings specified in this paper. 
erations, radiation treatment, and chemotherapy) associated with neurocognitive outcome among these children. $1,8,9,11,12,14-16,21-23,29$

We previously reported that among patients with serum sodium concentrations $\leq 130 \mathrm{mEq} / \mathrm{L}$ following initial surgical care for pediatric brain tumors, $21 \%$ of patients had hyponatremic seizures and $41 \%$ had altered mental status documented by the care team. Furthermore, we found that any serum sodium concentration $\leq 130 \mathrm{mEq} / \mathrm{L}$ during the admission was independently and strongly associated with worse cognitive outcome. ${ }^{31}$ In fact, hyponatremia was associated with a more than 6-fold increased odds of moderate or worse disability when adjusted for age and tumor characteristics. ${ }^{31}$ However, aspects of hyponatremia such as rapidity of sodium concentration change and magnitude and duration of hyponatremia have not been evaluated in relation to patient outcomes.

In the current study, we sought to determine if aspects of sodium disruption were independently associated with neurocognitive outcome following surgery for pediatric intracranial neoplasms. We hypothesized that there would be an exposure-response relationship between the severity of hyponatremia and worse patient outcome. Documentation of such a relationship would enhance medical evidence that therapies aimed to decrease or prevent hyponatremia might improve neurocognitive outcome in pediatric survivors of intracranial neoplasms.

\section{Methods \\ Study Population}

We conducted a single-center retrospective cohort study of children aged 0-19 years with an intracranial neoplasm who were admitted for a first neurosurgical procedure. We have previously examined this cohort to report the incidence and risk factors for hyponatremia, the time course and severity of hyponatremia, and an exploratory association between serum sodium concentration $\leq 130$ $\mathrm{mEq} / \mathrm{L}$ and cognitive outcome. ${ }^{4,31}$ In this study we sought to assess risk factors for worse cognitive outcome and the relationship between hyponatremia severity and cognitive outcome. Patient cognitive outcomes were measured by Pediatric Cerebral Performance Category (PCPC) scores assigned at the time of hospital discharge. ${ }^{10}$ This study was approved by the University of Utah Institutional Review Board and granted a waiver of need for informed consent.

All patient medical records from Primary Children's Hospital in Salt Lake City, Utah, were reviewed for admissions between January 2001 and February 2012 with an International Classification of Diseases, Ninth Revision, Clinical Medicine (ICD-9-CM) discharge code for either malignant or benign intracranial neoplasm (ICD-9-CM codes 191.0-191.9 or 225.0-225.2) and a concurrent ICD9-CM procedure code for intracranial tumor resection or biopsy (ICD-9-CM codes 01.59 or $01.13-01.14$ ); primary pituitary tumors and craniopharyngiomas were not included in the cohort due to separate ICD-9-CM codes. Four hundred eighty-two encounters were initially identified among 372 unique patients; 6 of these encounters were duplicated. After identification with administrative data, an independent chart review was conducted. Three encounters were excluded because the patients did not undergo tumor resection or biopsy. For analysis, we selected patients who underwent only their first neurosurgical procedure for a tumor and required at least 1 serum sodium check during admission. Three hundred nineteen distinct patients and encounters were included in the final analysis.

\section{Data Collection}

Patients were stratified by cognitive outcome based on PCPC scores assigned from examinations at hospital discharge as follows: 1) normal, 2) mild disability, 3) moderate disability, 4) severe disability, 5) coma or vegetative state, and 6) brain death..$^{10}$ Mild disability was defined as able to interact at age-appropriate levels, attending regular school, and having a mild neurological deficit (e.g., cranial nerve palsy). Moderate disability was defined as neurological function sufficient to perform activities of daily living independently, a moderate neurological deficit (e.g., hemiparesis), and/or requiring special education. Severe disability was defined as requiring dependent care to perform activities of daily living due to impaired brain function. A dichotomized PCPC score was used to define poor outcome, corresponding to a PCPC score of 3-6 (moderate disability to brain death), compared with a PCPC score of 1-2 (normal to mild disability). PCPC scores were originally collected for the analysis regarding risk factors for hyponatremia. ${ }^{31}$ Although the authors were not blinded to sodium values, the original outcome was the presence of hyponatremia, not cognitive outcome, at the time of PCPC score data abstraction.

Demographic and clinical information were collected and compared among PCPC score groups. Tumor location, determined by radiological and surgical reports, was grouped among 4 regions for analysis: 1) cortical, 2) cerebellum and brainstem, 3) deep brain and ventricles, and 4) overlapping, which included tumors involving at least 2 of the 3 defined regions. Pathology and radiology reports were reviewed to determine tumor histology and presence of metastases or obstructive hydrocephalus.

Serum sodium values with collection dates and times throughout admission were collected on each patient. Mild hyponatremia was defined as any serum sodium value $\geq$ $131-135 \mathrm{mEq} / \mathrm{L}$ and severe hyponatremia was defined as any sodium value $\leq 130 \mathrm{mEq} / \mathrm{L}$ during the admission. The time at which low serum sodium values occurred for a given patient was used to review records for symptomatic hyponatremia, including hyponatremic seizures or altered mental status. Seizures or altered mental status were ascribed to hyponatremia only with clinical documentation from a medical provider who cared for the patient and attributed the symptom to hyponatremia, and a concurrent serum sodium concentration $\leq 130 \mathrm{mEq} / \mathrm{L}$ within 1 hour of the documented symptom. Treatments for hyponatremia included fluid restriction, normal saline fluid bolus, hypertonic saline infusion, and enteral sodium salt supplementation.

Nadir sodium was the lowest serum sodium concentration measured during admission. Rapid changes in sodium concentration were assessed as decreases $>10$ or $>$ $15 \mathrm{mEq} / \mathrm{L}$ within any 24-hour period of admission. An episode of severe or mild hyponatremia was defined as a 
distinct period of low sodium below the assigned thresholds with sodium rising above the thresholds to terminate an episode. Subsequent episodes were recorded if sodium concentration again fell below the assigned thresholds. The total duration of severe or mild hyponatremia was determined by adding together the lengths of each defined episode (onset to termination in days).

\section{Statistical Analyses}

All laboratory and collected demographic and clinical information were entered into a database using the OpenClinica program (www.openclinica.com, Isovera, Inc.). Analyses were then conducted using $\mathrm{R}$ software (version 2.15.2, www.r-project.com). Univariate results were reported as counts and percentages for categorical variables and as medians with interquartile ranges (IQRs) for continuous variables. The relationship between demographic, clinical, and hyponatremia variables and worse cognitive outcome at discharge was explored by assessing PCPC score as a graduated outcome (score 1-6) in bivariate analyses using a permutation version of the Cochran-Armitage trend test (categorical) and Spearman's correlation (continuous). All tests were 2-tailed and significance was defined as $p<0.05$.

Several multivariable models were created to explore the association of hyponatremia variables with odds of poor outcome (dichotomized PCPC score 3-6 vs 1-2). Sodium concentration nadir, rates of sodium change, duration of hyponatremia, and episodes of hyponatremia were all individually adjusted for patient factors (age, tumor location, malignant histology, and hydrocephalus) previously associated with worsened neurocognitive outcomes. ${ }^{1,8,9,11 \text {, }}$ 12,14,15,21-23,29 A multivariable model was also created containing the same patient factors and measures of sodium derangement (sodium concentration nadir, sodium value decrease $>15 \mathrm{mEq} / \mathrm{L}$ in 24 hours, duration of mild hyponatremia, episodes of mild hyponatremia) to determine which aspects of sodium disruption remained independently associated with poor outcome. Forward and backward stepwise selection using the Akaike Information Criteria was used to present a reduced, best-fit model. Multicollinearity was evaluated among predictor variables in the presented models using the variance inflation factor (VIF). We used VIF $>10$ as a guide for exploring alternative models. Results of multivariable analyses were reported as an adjusted odds ratio (OR) with a 95\% confidence interval (CI). The adjusted OR and 95\% CI for variables in the reduced multivariable model were used to calculate predicted probabilities of poor outcome for sodium concentration nadir while holding duration of mild hyponatremia at mean values when stratifying by tumor region.

\section{Results}

Three hundred nineteen patients undergoing a first neurosurgery for an intracranial tumor were identified. Table 1 presents patient demographic and clinical characteristics by cognitive outcome assessed with PCPC score at discharge. Forty-three percent of the patients $(n=137)$ were categorized as cognitively normal at hospital discharge and 32\% ( $\mathrm{n}=102)$ had only mild disability. Twenty-five percent $(n=80)$ had poor outcome (PCPC score 3-6) at hospital discharge. Two patients died during admission: 1 met the criteria for neurological determination of death and the other was classified as in a persistent vegetative state by the final documented neurological examination prior to death. Sixty-five patients $(20 \%)$ had moderate disability and 13 (4\%) were categorized as having severe disability at discharge. Worse cognitive outcomes evidenced by increasing PCPC scores at discharge were significantly associated with younger age, obstructive hydrocephalus, and noncortical tumor location (all $\mathrm{p}<0.05$ ). As expected, increased PCPC score was also associated with longer length of stay ( $\mathrm{p}<0.001)$. One hundred thirty-seven children $(43 \%)$ had serum sodium concentrations $\geq 131-135$ $\mathrm{mEq} / \mathrm{L}$, with 39 (12\%) developing severe hyponatremia, which was significantly associated with worse PCPC scores $(\mathrm{p}<0.001)$. Symptomatic hyponatremia, including hyponatremic seizures, was also associated with worse PCPC score at discharge $(\mathrm{p}<0.001)$. Similarly, receiving at least 1 hyponatremia treatment was associated with worse PCPC score $(p<0.001)$. Eighty-seven percent of patients with severe hyponatremia and $27 \%$ of patients with mild hyponatremia received a treatment. Although prescribed care varied, all patients with symptomatic hyponatremia were treated and all patients with hyponatremic seizures received hypertonic saline infusion.

Table 2 presents information regarding sodium concentration, variability, and duration of hyponatremia across PCPC scores. Rapid decreases in sodium were uncommon overall $(n=30)$, but more than $30 \%$ of patients with severe disability at discharge had at least 1 episode of sodium concentration decreasing $>15 \mathrm{mEq} / \mathrm{L}$ within a 24-hour period. Additionally, nadir sodium concentration during admission was significantly lower among children with worse PCPC scores $(\mathrm{p}<0.001)$. Among patients with moderate disability by PCPC score, $25 \%$ had nadir sodium values below $130 \mathrm{mEq} / \mathrm{L}$. Among patients with severe disability, 25\% had nadir sodium values below $123 \mathrm{mEq} / \mathrm{L}$.

Some patients had a single episode of hyponatremia and spent little time with abnormal sodium values during admission. However, as noted in Table 2, patients with worse PCPC scores at discharge had significantly more episodes of hyponatremia and accumulated significantly more days with mild and severe hyponatremia (all $\mathrm{p}<$ 0.001 ). Twenty-five percent of patients with severe disability had more than 9 unique episodes of mild hyponatremia and spent more than 16 accumulated admission days with at least mild hyponatremia. Episodes of severe hyponatremia were less common overall, but $25 \%$ of patients with severe disability had more than 5 episodes and more than 2 accumulated admission days with severe hyponatremia.

Table 3 presents the multivariable analysis for measures of sodium derangement individually adjusted for patient factors previously associated with poor outcome (age, obstructive hydrocephalus, tumor location, and malignant histology). ${ }^{1,8,9,11,12,14,15,21-23,29}$ Rapid declines in serum sodium $>15 \mathrm{mEq} / \mathrm{L}$ within 24 hours, nadir sodium concentration, duration of hyponatremia, and number of episodes of hyponatremia all remained independently associated with increased odds of poor outcome.

Given the interrelated nature of some variables, such as duration and number of episodes of hyponatremia, a full 
TABLE 1. Select demographic and clinical information stratified by PCPC score

\begin{tabular}{|c|c|c|c|c|c|c|c|}
\hline \multirow[b]{2}{*}{ Variable } & \multicolumn{6}{|c|}{ PCPC Score* } & \multirow{2}{*}{$\begin{array}{c}p \text { Value } \\
\text { (correlation coefficient) } \dagger\end{array}$} \\
\hline & 1 & 2 & 3 & 4 & 5 & 6 & \\
\hline No. of patients & 137 & 102 & 65 & 13 & 1 & 1 & \\
\hline Median age in yrs (IQR) & $7(3-12)$ & $8(4-11)$ & $5(2-8)$ & $5(1-10)$ & 2 & 0 & $0.04(-0.11)$ \\
\hline Males (\%) & $73(53)$ & $56(55)$ & $38(58)$ & $10(77)$ & $1(100)$ & 0 & 0.24 \\
\hline Resection performed (\%) & $118(86)$ & $86(84)$ & $61(94)$ & $12(92)$ & $1(100)$ & $1(100)$ & 0.18 \\
\hline Race $(\%)$ & & & & & & & 0.67 \\
\hline Caucasian & $120(88)$ & $88(86)$ & $57(88)$ & $11(85)$ & $1(100)$ & $1(100)$ & \\
\hline Hispanic & $12(9)$ & $12(12)$ & $7(11)$ & $2(15)$ & 0 & 0 & \\
\hline Other & $5(3)$ & $2(2)$ & $1(1)$ & 0 & 0 & 0 & \\
\hline Median days of admission (IQR) & $5.7(4.1-8.4)$ & $7.3(5.4-10.4)$ & $13.9(9.2-24.5)$ & $29(15.9-49.3)$ & 4.3 & 1.9 & $<0.001(0.47)$ \\
\hline Malignant histology (\%) & $70(51)$ & $51(50)$ & $37(57)$ & $9(69)$ & $1(100)$ & $1(100)$ & 0.14 \\
\hline Metastases (\%) & $14(10)$ & $9(9)$ & $8(12)$ & $3(23)$ & 0 & $1(100)$ & 0.12 \\
\hline Obstructive hydrocephalus (\%) & $72(53)$ & $64(63)$ & $48(74)$ & $10(77)$ & $1(100)$ & $1(100)$ & $<0.001$ \\
\hline Tumor location (\%) & & & & & & & $<0.001$ \\
\hline Cortical & $39(28)$ & $16(16)$ & $6(9)$ & 0 & 0 & 0 & \\
\hline Cerebellum or brainstem & $37(27)$ & $38(37)$ & $18(28)$ & $5(38)$ & 0 & $1(100)$ & \\
\hline Deep brain or ventricles & $48(35)$ & $33(32)$ & $27(42)$ & $5(38)$ & 0 & 0 & \\
\hline Overlapping & $13(10)$ & $15(15)$ & $14(22)$ & $3(23)$ & $1(100)$ & 0 & \\
\hline Any hyponatremia (\%) & $62(45)$ & $55(54)$ & $47(72)$ & $10(77)$ & $1(100)$ & $1(100)$ & $<0.001$ \\
\hline Severe hyponatremia & $6(4)$ & $8(8)$ & $17(26)$ & $7(54)$ & $1(100)$ & 0 & $<0.001$ \\
\hline Symptomatic hyponatremia (\%) & $5(4)$ & $4(4)$ & $12(18)$ & $5(38)$ & 0 & 0 & $<0.001$ \\
\hline Hyponatremic seizure & $1(<1)$ & $1(1)$ & $2(3)$ & $5(38)$ & 0 & 0 & $<0.001$ \\
\hline Treatment for hyponatremia (\%) & $16(12)$ & $20(20)$ & $27(42)$ & $8(62)$ & $1(100)$ & 0 & $<0.001$ \\
\hline
\end{tabular}

* PCPC scores: 1 = normal, 2 = mild disability, $3=$ moderate disability, $4=$ severe disability, $5=$ coma/vegetative, 6 = brain death.

$\dagger$ Continuous variables were assessed with Spearman's correlation coefficient and reported with $p$ values and correlation coefficients, whereas categorical variables were assessed using the Cochran-Armitage trend test and reported with $p$ values.

multivariable model with measures of sodium derangement as well as patient factors is presented in Table 4. In spite of the interrelatedness of some variables, our multicollinearity check did not reveal any problems, with all predictor variables in the full and reduced models having VIFs < 3. After stepwise selection, the reduced, best-fit multivariable model showed nadir sodium (adjusted OR $0.86,95 \%$ CI $0.78-0.95$ ) and noncortical tumor locations remained important independent risk factors for poor outcome (Table 4). Figure 1 presents the predicted probability of poor outcome by nadir sodium concentration for individual tumor regions as calculated from the final reduced multivariable model. For all noncortical tumor locations, a decrease in nadir sodium value of $10 \mathrm{mEq} / \mathrm{L}$ below normal laboratory values was associated with a greater than $25 \%$ increase in the predicted probability of poor outcome.

\section{Discussion}

A quarter of the children in this study had a poor outcome following intracranial tumor surgery. Young age, hydrocephalus, noncortical tumor location, and hyponatremia were significantly associated with worsening cognitive function. The severity of hyponatremia, measured by the presence of symptoms, requiring treatment, lower sodium nadir concentration, and longer duration of hypo- natremia were all significantly associated with worsening PCPC scores. Increased sodium variability (rapid decreases and more distinct episodes of hyponatremia) was also significantly associated with worse outcome. When these interrelated aspects of hyponatremia were tested controlling for patient and tumor factors, noncortical tumor locations and nadir sodium concentration remained important independent risk factors associated with poor cognitive outcome at hospital discharge.

More than half of the children treated for brain tumors $(57 \%)$ had some disability, with a quarter having moderate $(21 \%)$ or severe $(4 \%)$ disability at discharge, consistent with previous studies of functional and neurocognitive outcomes in children with brain tumors. 17,17,21,22,27,32 Macedoni-Luksic et al. reported $62 \%$ of children with brain tumors had at least 1 neurological impairment, with $22 \%$ moderately disabled and $2 \%$ severely disabled more than 3 years after treatment. ${ }^{17}$ Sønderkaer et al. reported $69 \%$ of patients with brain tumors had a neurological deficit approximately 10 years from treatment, and using the Bloom's score, which closely mirrors aspects of the PCPC score, they estimated $23 \%$ had moderate disability and $2 \%$ had severe disability. ${ }^{27}$ The similarities among cognitive outcomes were consistent despite variation in length of follow-up and measures of outcome (i.e., Bloom's score, intellectual quotient, and parent/patient questionnaire). 


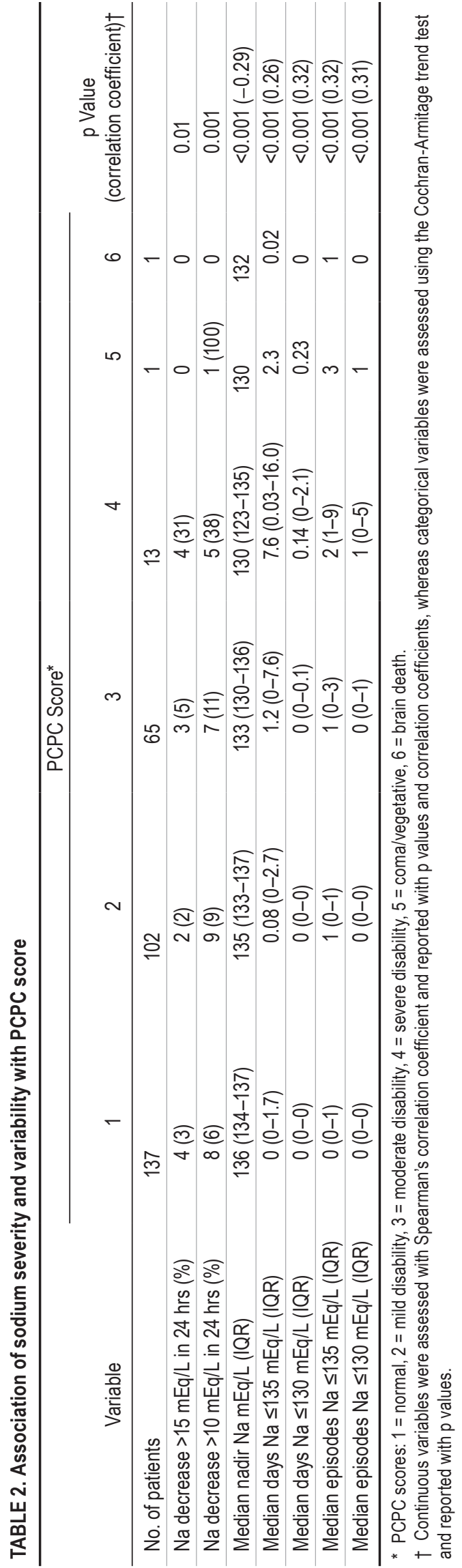

TABLE 3. Multivariable adjusted sodium variables as predictors of poor outcome

\begin{tabular}{lc}
\hline \multicolumn{1}{c}{ Factor $^{*}$} & Adjusted OR $(95 \% \mathrm{Cl})$ \\
\hline$>15 \mathrm{mEq} / \mathrm{L}$ decrease in Na w/in 24 hrs & $3.45(1.07-11.31)$ \\
\hline$>10 \mathrm{mEq} / \mathrm{L}$ decrease in Na w/in 24 hrs & $2.16(0.97-4.81)$ \\
\hline Nadir Na during admission (mEq/L) & $0.83(0.76-0.89)$ \\
\hline Duration serum Na $\leq 135 \mathrm{mEq} / \mathrm{L}$ (days) & $1.12(1.06-1.18)$ \\
\hline Duration serum $\mathrm{Na} \leq 130 \mathrm{mEq} / \mathrm{L}$ (days) & $2.05(1.18-3.57)$ \\
\hline Unique episodes of $\mathrm{Na} \leq 135 \mathrm{mEq} / \mathrm{L}$ & $1.23(1.09-1.37)$ \\
\hline Unique episodes of $\mathrm{Na} \leq 130 \mathrm{mEq} / \mathrm{L}$ & $1.40(1.11-1.76)$ \\
\hline
\end{tabular}

* All factors run in separate models controlling for age, tumor location, malignant histology, and obstructive hydrocephalus. Poor outcome defined as PCPC score of 3-6 at hospital discharge compared with scores of 1-2.

PCPC has been used to assess cognitive function of pediatric patients surviving intensive care and was validated with intelligence quotient scores among those children. ${ }^{10}$ The PCPC score mirrors measurements contained in the Bloom's score used for brain tumor survivors. ${ }^{21,27}$

We assessed cognitive function at initial hospital discharge with the PCPC score, which enabled assessment of all patients and limited the number of confounding factors, such as radiation, chemotherapy, and reoperations associated with worsened outcome with later followup. $1,9,14,16,21$ Both tumor progression and additional therapies (i.e., cranial radiation) cause cognitive dysfunction

TABLE 4. Full and reduced multivariable models predicting poor outcome adjusted for sodium variables*

\begin{tabular}{lc}
\hline \multicolumn{1}{c}{ Variable } & Adjusted OR $(95 \% \mathrm{Cl})$ \\
\hline Full model & $0.98(0.92-1.04)$ \\
\hline Age $(\mathrm{yrs})$ & $1.02(0.88-1.17)$ \\
\hline Unique episodes serum Na $\leq 135 \mathrm{mEq} / \mathrm{L}$ & $1.05(0.97-1.13)$ \\
\hline Duration serum Na $\leq 135 \mathrm{mEq} / \mathrm{L}$ (days) & $0.87(0.79-0.97)$ \\
\hline Nadir Na during admission (mEq/L) & $1.62(0.42-6.24)$ \\
\hline$>15 \mathrm{mEq} / \mathrm{L}$ decrease in Na w/in $24 \mathrm{hrs}$ & Reference \\
\hline Tumor location & $2.92(1.00-8.51)$ \\
\hline \multicolumn{1}{c}{ Cortex } & $2.89(0.99-8.44)$ \\
\hline Cerebellum or brainstem & $5.23(1.66-16.43)$ \\
\hline Deep brain or ventricles & $0.89(0.46-1.73)$ \\
\hline Overlapping & $1.00(0.55-1.79)$ \\
\hline Obstructive hydrocephalus & \\
\hline Malignant histology & $0.86(0.78-0.95)$ \\
\hline Reduced model after stepwise selection & $1.05(0.98-1.12)$ \\
\hline Nadir Na during admission (mEq/L) & \\
\hline Duration serum Na $\leq 135 \mathrm{mEq} / \mathrm{L}$ (days) & Reference \\
\hline Tumor location & $2.97(1.08-8.18)$ \\
\hline Cortex & $2.98(1.10-8.04)$ \\
\hline Cerebellum or brainstem & $5.41(1.83-16.02)$ \\
\hline Deep brain or ventricles & \\
\hline Overlapping &
\end{tabular}

* Poor outcome defined as PCPC score of 3-6 at hospital discharge compared to scores of $1-2$. 


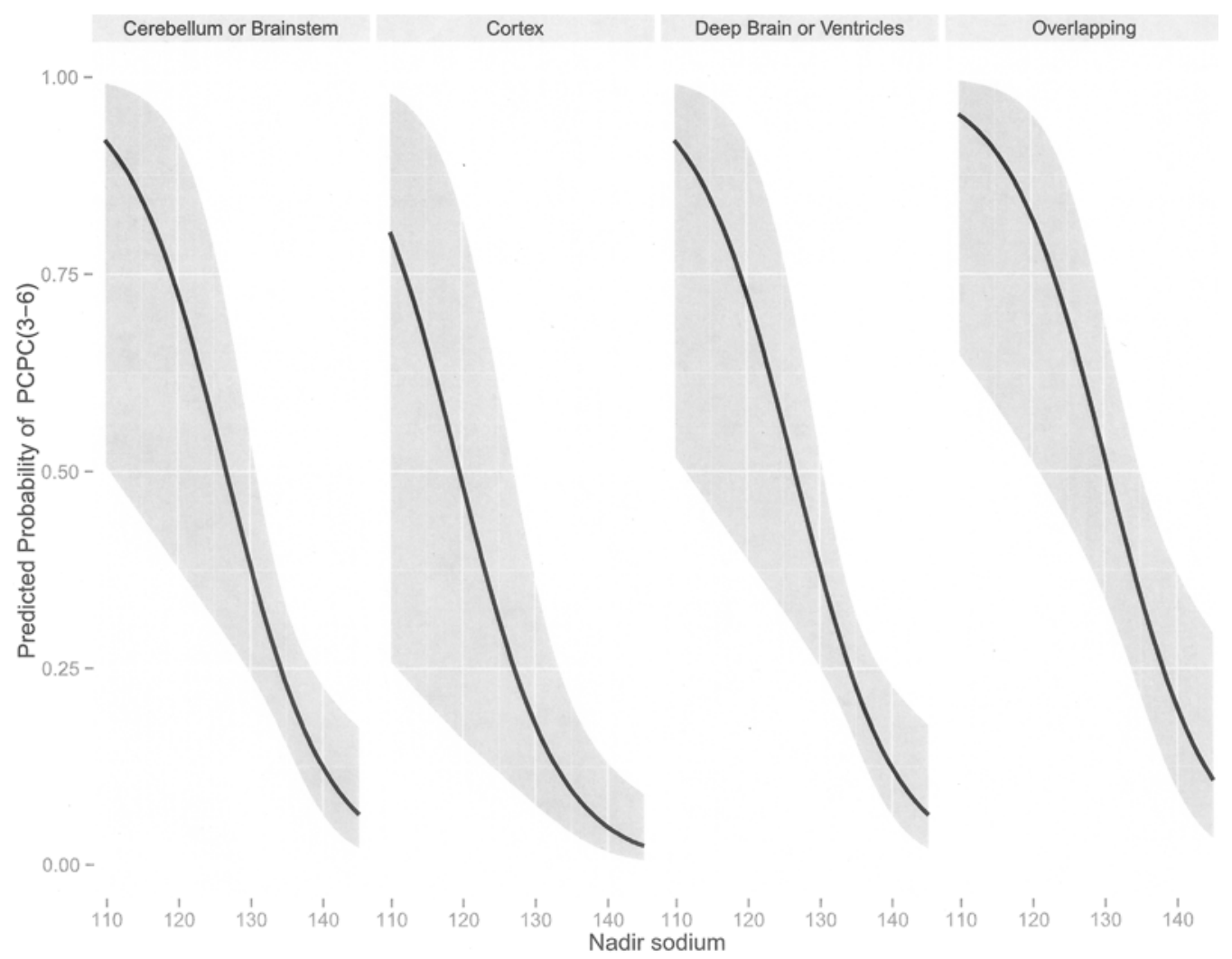

FIG. 1. Graph of the predicted probabilities calculated from adjusted ORs (solid lines) and $95 \% \mathrm{Cls}$ (shaded regions) from the reduced, best-fit multivariable model for poor outcome (PCPC score 3-6). Values for nadir sodium concentration across the 4 tumor regions presented while holding duration of sodium $\leq 135 \mathrm{mEq} / \mathrm{L}$ at mean values.

with later onset and progression over time. ${ }^{1,9,16}$ Neurosurgical procedures themselves worsen neurological status in approximately one-third of patients, $, 3,27$ and patients needing reoperations have worsened long-term outcomes..$^{21,29}$ Additionally, Sønderkaer et al. ${ }^{27}$ reported $38 \%$ of patients with surgically treated brain tumors had new deficits postoperatively. Moderate and severe disabilities were likely to persist from the postoperative period on long-term followup, whereas some mild disabilities, such as cranial nerve palsies, improved.$^{27}$ This tendency for persistence of substantial deficits may account for some of the similarities in the incidence of moderate and severe disabilities noted in our study at discharge and in other studies over a wider interval of follow-up.

Our previous study found a 6-fold increased odds of poor outcome with any occurrence of severe hyponatremia during surgical admissions for pediatric brain tumors. ${ }^{31}$ The current study is the first to evaluate the associations between severity of hyponatremia and neurocognitive outcome in this population. However, hyponatremia has been associated with worsened morbidity and mortality in a variety of adult and pediatric populations. Among adult patients, hyponatremia is associated with increased mortality in adult surgical intensive care patients, ${ }^{24}$ increased complications among patients with heart failure, ${ }^{3}$ and worse Glasgow Outcome Scale score following subarachnoid hemorrhage ${ }^{25}$ and traumatic brain injury. ${ }^{20}$ Among pediatric populations, hyponatremia is associated with increased mortality among liver transplant patients ${ }^{5}$ and in children with pneumococcal meningitis. ${ }^{6}$ Al-Zahraa Omar and Al Bunyan described 72 children with a variety of neurological diagnoses, including 2 patients with a brain tumor, and noted hyponatremia was associated with an increased length of stay compared with patients with sodium concentrations greater than $130 \mathrm{mEq} / \mathrm{L}$. Furthermore, they reported worse neurological disability among those with more severe hyponatremia. ${ }^{2}$

Hyponatremia is common following pediatric brain tumor surgeries $\mathrm{s}^{30,31}$ and is caused by a variety of mechanisms including cerebral salt wasting, syndrome of inappropriate secretion of antidiuretic hormone, medications, and intravenous fluids. ${ }^{26}$ Hyponatremia from any mechanism can cause or exacerbate neurological injury with seizures, encephalopathy, and cerebral edema. ${ }^{19,26,28}$ Twenty-six patients $(8 \%)$ met our definition of symptomatic hyponatremia, including $9(3 \%)$ with hyponatremic seizures, and this was associated with worsened outcome in our cohort. Increased susceptibility to hyponatremic encephalopathy and neurological deterioration with hyponatremia complicates a variety of pediatric neurological diseases. ${ }^{18,19}$ Despite widespread association of hyponatremia with worse outcomes and a potential mechanism for brain injury due to osmolar shifts with hyponatremia, we cannot conclude there is a causal association between hyponatremia and worse outcome with any retrospective analysis. However, given the potential for secondary injury by hyponatremia, the strong association with worse outcome in this and other studies, and the positive exposure-response relationship 
between severity of sodium derangement and outcome noted in this study, close attention to sodium values in this population is warranted.

Young age, hydrocephalus, tumor location, and malignant histology are interrelated factors associated with worsened neurological deficits among pediatric brain tumor survivors. 1,8,,11,12,14,15,21-23,29 These same factors were also associated with risk of developing hyponatremia in the postoperative period, ${ }^{30,31}$ but previous studies of neurocognitive outcomes have not evaluated hyponatremia alone or in association with these factors. We found that young patient age and hydrocephalus were associated with worsened graduated PCPC score but did not remain independent risk factors for poor outcome in multivariable analysis, possibly due to interrelatedness with hyponatremia and tumor location. Malignant histology was not significantly associated with outcome in this study, but patients with malignant tumors will likely undergo more therapeutic interventions, including reoperation and radiation, that may worsen cognitive function over time. Noncortical tumor locations remained an important independent risk factor for poor outcome in our study. Variation in the literature exists regarding the effects of tumor location on outcome given that many studies focus on a particular tumor type (e.g., astrocytoma), anatomical location (e.g., posterior fossa), deficit (e.g., attention), or patient population (e.g., infants). ${ }^{1,8,22,23}$ The association between hyponatremia, tumor location, and outcome among these specific patient subgroups has not been studied.

Our study has limitations to consider. Given the retrospective nature of data collection, accuracy of some of the data cannot be confirmed and we cannot infer a causal relationship between exposures such as hyponatremia and neurological disability. We chose to limit confounding variables by measuring short-term neurocognitive outcome at hospital discharge. The presurgical cognitive status of the patients and whether postoperative deficits persist long-term was not evaluated, but is an important consideration in future studies. Additionally, given the retrospective nature of the study, multiple causes of hyponatremia, variability in treatment thresholds, and multiple types of treatment administered to our patients, we were unable to assess the effects of treatment or cause of hyponatremia on outcome. Whether early and proper intervention for hyponatremia improves outcome in these patients is an important question for future research.

\section{Conclusions}

Twenty-five percent of the children in this study had moderate or worse disabilities at hospital discharge following surgery for intracranial tumors. A strong association was found between increasing severity of hyponatremia and worsened cognitive outcome. Lower nadir sodium concentration and noncortical tumor locations were independently associated with poor outcome when adjusted for patient factors, tumor characteristics, and measures of sodium derangement. This study emphasizes the importance of monitoring serum sodium following pediatric brain tumor surgery given the potential of hyponatremia to exacerbate neurological injury. Future studies are needed to de- velop targeted protocols for hyponatremia to determine if intervention can improve outcome among these children.

\section{References}

1. Aarsen FK, Paquier PF, Arts WF, Van Veelen ML, Michiels E, Lequin M, et al: Cognitive deficits and predictors 3 years after diagnosis of a pilocytic astrocy toma in childhood. $\mathbf{J}$ Clin Oncol 27:3526-3532, 2009

2. Al-Zahraa Omar F, Al Bunyan M: Severe hyponatremia as poor prognostic factor in childhood neurologic diseases. J Neurol Sci 151:213-216, 1997

3. Arévalo Lorido JC, Carretero Gómez J, Formiga F, Montero Pérez-Barquero M, Trullás Vila JC, Aramburu Bodas O, et al: Hyponatremia as predictor of worse outcome in real world patients admitted with acute heart failure. Cardiol J 20:506512, 2013

4. Belzer JS, Williams CN, Riva-Cambrin J, Presson AP, Bratton SL: Timing, duration, and severity of hyponatremia following pediatric brain tumor surgery. Pediatr Crit Care Med 15:456-463, 2014

5. Carey RG, Bucuvalas JC, Balistreri WF, Nick TG, Ryckman FR, Yazigi N: Hyponatremia increases mortality in pediatric patients listed for liver transplantation. Pediatr Transplant 14:115-120, 2010

6. Chao YN, Chiu NC, Huang FY: Clinical features and prognostic factors in childhood pneumococcal meningitis. J Microbiol Immunol Infect 41:48-53, 2008

7. Daszkiewicz P, Maryniak A, Roszkowski M, Barszcz S: Long-term functional outcome of surgical treatment of juvenile pilocytic astrocytoma of the cerebellum in children. Childs Nerv Syst 25:855-860, 2009

8. Di Rocco C, Chieffo D, Pettorini BL, Massimi L, Caldarelli M, Tamburrini G: Preoperative and postoperative neurological, neuropsychological and behavioral impairment in children with posterior cranial fossa astrocytomas and medulloblastomas: the role of the tumor and the impact of the surgical treatment. Childs Nerv Syst 26:1173-1188, 2010

9. Ellenberg L, Liu Q, Gioia G, Yasui Y, Packer RJ, Mertens A, et al: Neurocognitive status in long-term survivors of childhood CNS malignancies: a report from the Childhood Cancer Survivor Study. Neuropsychology 23:705-717, 2009

10. Fiser DH, Long N, Roberson PK, Hefley G, Zolten K, BrodieFowler M: Relationship of pediatric overall performance category and pediatric cerebral performance category scores at pediatric intensive care unit discharge with outcome measures collected at hospital discharge and 1- and 6-month follow-up assessments. Crit Care Med 28:2616-2620, 2000

11. Gragert MN, Ris MD: Neuropsychological late effects and rehabilitation following pediatric brain tumor. J Pediatr Rehabil Med 4:47-58, 2011

12. Hardy KK, Bonner MJ, Willard VW, Watral MA, Gururangan S: Hydrocephalus as a possible additional contributor to cognitive outcome in survivors of pediatric medulloblastoma. Psychooncology 17:1157-1161, 2008

13. Houdemont SP, De Carli E, Delion M, Ringuier B, Chapotte C, Jeudy C, et al: Short-term neurological outcome of children after surgery for brain tumors: incidence and characteristics in a pediatric intensive care unit. Childs Nerv Syst 27:933-941, 2011

14. Huber JF, Bradley K, Spiegler B, Dennis M: Long-term neuromotor speech deficits in survivors of childhood posterior fossa tumors: effects of tumor type, radiation, age at diagnosis, and survival years. J Child Neurol 22:848-854, 2007

15. Kulkarni AV, Piscione J, Shams I, Bouffet E: Long-term quality of life in children treated for posterior fossa brain tumors. J Neurosurg Pediatr 12:235-240, 2013

16. Mabbott DJ, Penkman L, Witol A, Strother D, Bouffet E: Core neurocognitive functions in children treated for posterior fossa tumors. Neuropsychology 22:159-168, 2008 
17. Macedoni-Luksic M, Jereb B, Todorovski L: Long-term sequelae in children treated for brain tumors: impairments, disability, and handicap. Pediatr Hematol Oncol 20:89-101, 2003

18. McJunkin JE, de los Reyes EC, Irazuzta JE, Caceres MJ, Khan RR, Minnich LL, et al: La Crosse encephalitis in children. N Engl J Med 344:801-807, 2001

19. Moritz ML, Ayus JC: New aspects in the pathogenesis, prevention, and treatment of hyponatremic encephalopathy in children. Pediatr Nephrol 25:1225-1238, 2010

20. Moro N, Katayama Y, Igarashi T, Mori T, Kawamata T, Kojima J: Hyponatremia in patients with traumatic brain injury: incidence, mechanism, and response to sodium supplementation or retention therapy with hydrocortisone. Surg Neurol 68:387-393, 2007

21. Pietilä S, Korpela R, Lenko HL, Haapasalo H, Alalantela R, Nieminen P, et al: Neurological outcome of childhood brain tumor survivors. J Neurooncol 108:153-161, 2012

22. Pillai S, Metrie M, Dunham C, Sargent M, Hukin J, Steinbok P: Intracranial tumors in infants: long-term functional outcome, survival, and its predictors. Childs Nerv Syst 28:547555,2012

23. Ris MD, Beebe DW, Armstrong FD, Fontanesi J, Holmes E, Sanford RA, et al: Cognitive and adaptive outcome in extracerebellar low-grade brain tumors in children: a report from the Children's Oncology Group. J Clin Oncol 26:4765-4770, 2008

24. Sakr Y, Rother S, Ferreira AM, Ewald C, Dünisch P, Riedemmann N, et al: Fluctuations in serum sodium level are associated with an increased risk of death in surgical ICU patients. Crit Care Med 41:133-142, 2013

25. Saramma P, Menon RG, Srivastava A, Sarma PS: Hyponatremia after aneurysmal subarachnoid hemorrhage: implications and outcomes. J Neurosci Rural Pract 4:24-28, 2013

26. Smith DM, McKenna K, Thompson CJ: Hyponatraemia. Clin Endocrinol (Oxf) 52:667-678, 2000

27. Sønderkaer S, Schmiegelow M, Carstensen H, Nielsen LB, Müller J, Schmiegelow K: Long-term neurological outcome of childhood brain tumors treated by surgery only. J Clin Oncol 21:1347-1351, 2003

28. Sterns RH, Hix JK, Silver SM: Management of hyponatremia in the ICU. Chest 144:672-679, 2013
29. Vinchon M, Baroncini M, Leblond P, Delestret I: Morbidity and tumor-related mortality among adult survivors of pediatric brain tumors: a review. Childs Nerv Syst 27:697-704, 2011

30. Williams C, Simon TD, Riva-Cambrin J, Bratton SL: Hyponatremia with intracranial malignant tumor resection in children. J Neurosurg Pediatr 9:524-529, 2012

31. Williams CN, Belzer JS, Riva-Cambrin J, Presson AP, Bratton SL: The incidence of postoperative hyponatremia and associated neurological sequelae in children with intracranial neoplasms. J Neurosurg Pediatr 13:283-290, 2014

32. Wilne S, Koller K, Collier J, Kennedy C, Grundy R, Walker $\mathrm{D}$ : The diagnosis of brain tumours in children: a guideline to assist healthcare professionals in the assessment of children who may have a brain tumour. Arch Dis Child 95:534-539, 2010

\section{Author Contributions}

Conception and design: Williams, Riva-Cambrin, Bratton. Acquisition of data: Williams. Analysis and interpretation of data: all authors. Drafting the article: Williams. Critically revising the article: all authors. Reviewed submitted version of manuscript: all authors. Approved the final version of the manuscript on behalf of all authors: Williams. Statistical analysis: Williams, Presson, Bratton. Administrative/technical/material support: Riva-Cambrin, Presson. Study supervision: Bratton.

\section{Supplemental Information}

\section{Previous Presentation}

The abstract of this work was presented in poster form at the Society for Critical Care Medicine national meeting on January 10, 2014, in San Francisco, California.

\section{Correspondence}

Cydni N. Williams, Oregon Health \& Science University, Pediatric Critical Care, 707 S.W. Gaines St., CDRC-P, Portland, OR 97239. email: willicyd@ohsu.edu. 